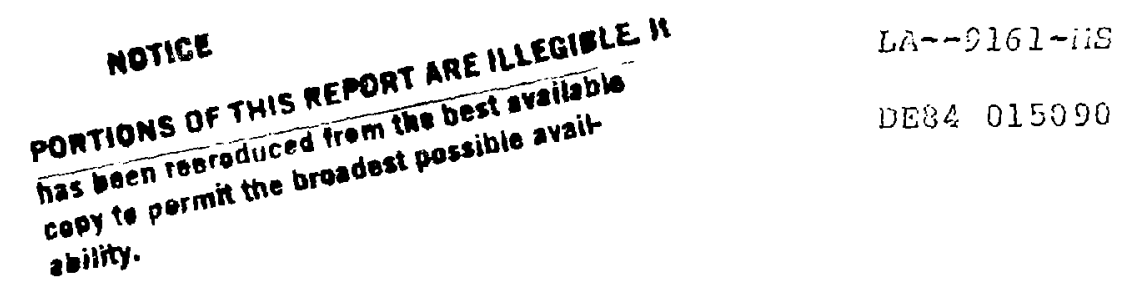

\title{
Oscillating Field Current Drive for Reversed Field Pinch Discharges
}

\author{
Kurt F. Schoenberg \\ Robert F. Gribble \\ Don A. Baker
}

\section{DISCLAIMER}

This report was prepared as an account of work sponsored by an agency of the United States Government Neither the United States Government nor any agency thereof, nor any of their employees, makes any warranty, express or implied, or assumes any legal liability or responsibility for the accuracy, completeness, or usefulness of any information, apparatus, product, or process disclosed, or represents that its use would not infringe privately owned rights. Refer. ence therein to any specific commercial praduct, process. or service by trade name, trademark, manufacturer, or otherwise does not necessarily consticute or imply its endorsement, recommendation, or fayoring by the United States Government or any agency thereof. The views and opinions of authors expressed herein do noi niccssarily state or reflect those of the United States Government or any agency thereor. 
14. K. F. Schoenberg,

C. J. Puchenauer,

R. S. Massey,

J. G. Melton, R. W. Moses, Jr., R. A. Nebsl, and J. A. Phillips, Phys. Fluids 27(3), 548 (1984).

15. D. A. Ehst, J. N. Brooks, T. Cha, K. Evans, Jr., A. Hassancin, S. Kim, S. Majumdar, B. Misra, and H. F. Stevens, "Tokamak Burn Cycle Study: A Duty Base for Comparing Long Pulse and Steady-State Power Reactors," Argonne National Laboratory report. ANL/FPP/TM-178, pp. 6-19 (1983).

16. E. J. Caramana and D. A. Baker, Nucl. Fuslon 24, 423 (1984).

17. D. A. Baker, Magnet1c Reconnection in Space and Laboratory Plasmas, Vol 30 of AGU Geomonograph Serjes, E. W. Hones, Jr., Ed. (American Geophysical Union, Washington, 1984 ).

18. M. G. Rushbridge, Plaswa Phys. 19, 499 (1977).

19. A. R. Jacobson and R. W. Moses, Jr., "Nonlocal DC Electrical Conductivity of a Lorentz Plasma in a Stochastic Magnetic Field," Los Alamos National Laboratory document LA-UR-83-3440, to be published in Phys. Rev. 
OSCILLATING FIELD CURRENT DRIVE FOR REVERSED FIELD FINCH DISCHARGES*

by

Kurt F. Schoenberg, Robert F. Gribble, and Don A. Baker

\begin{abstract}
Oscillating Field Current Drive (OFCD), also known as $F-\theta$ pumping, is a steady-state current-drive technique proposed for the Reversed Field Pinch (RFP). Unlike other current-drive techniques, which employ hightechnology, invasive, and power intensive schemes using radio frequency or neutral particle injection, $F-\theta$ pumping encails driving the toroidal and poloidal magnetic field circuits with low-freqiency (audio) oscillating voltage sources. Currert drive by this technique is a conseyuence of the strong nonlinear plasma coupling in the RFP. Because of its low frequency and efficient plasma coupling, $F-\theta$ pumping shows excellent promise as a reactor-relevant current-drive technique. A conceptual and computational study of this concept, including its experimental and reactor relevance, is explored in this paper.
\end{abstract}

\title{
I. INTRODUCTION
}

A variety of techniques has been proposed to drive steady-state current in toroidal containment devices.l To a large extent, the techniques employ high technology, invasive, power intensive schemes utilizing radio frequency or neutral particle injection. For large-scale, systems, the engineering and economic designs of these schemes are complex.

The dc drive situation for a Reversed Field Pinch (RFP) may be different. In an RFP, the poloidal and torolda: winding currents are nonlinearly coupled by the plasma. As a consequence, with certain circuit programing, the possibility

\footnotetext{
* This is a revised and updated version of a manuscript submitted December 10 , 1981. This report also forms the basis of a manuscript to be published in the Journal of Applied Physics in Vol. 55, September 1984.
} 
arises of maintaining a mean (time-averaged) toroldal current against dissipation without a continuous expenditure of mean magnetizing (or driving) flix. ${ }^{2}$ Thus, the promise exists for a relatively simple, steady-state currentdrive system that entails driving the toroidal and poloidal magnetic fiald circuits with low-frequency (audio) oscillating voltage sources. In addition, this oscillating Field Current Drive (OFCD), also known as $F-\theta$ pumping, shows excellent potential as a reactor-relevant current-drive technique. ${ }^{3}$ A conceptual study of OFCD w111 be explored in this paper.

II. A REVIEW OF DIRECT-CURRENT PRODUCTION FROM AN ALTERNATING-CURRENTDRIVEN NONLINEAR CIRCUIT

One normally considers the production of direct current from an ac voltage source in terms of a rectiffer. For 1llustrative purposes, we shall begin with a more general nonlinear single-port system, which includes the rectifier, and then proceed to a two-port system suitable for representing the RFP.

A. The One-Port System

In a on-port (one-terminal pair) system (Fig. 1), for a passive linear system, the steady-state current response to a sinusoldal voltage of radian frequency $\omega$ is given by

$$
I(\omega)=Y(\omega) V(\omega),
$$

where $V(\omega)=\hat{V} e^{I \omega t}, I(\omega)=\hat{I} e^{I \omega t}$, and $Y(\omega)$, called the complex driving point ac admittance, is the reciprocal of the complex impedance $z(\omega)$. As usual, the Instantaneous values of the voltage and current are represented by the real parts of $V(\omega) e^{I \omega t}$ and $I(\omega) e^{i \omega t}$, respectively. The relationship defined by

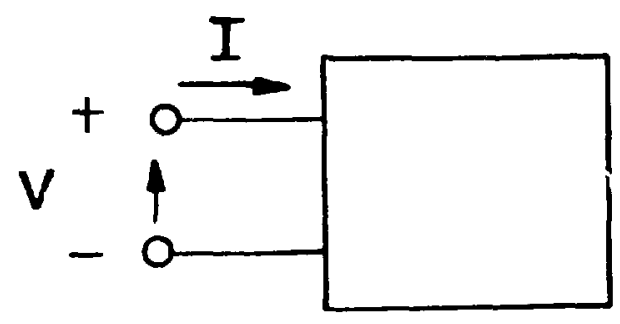

Fig. 1 . One-port system. 
Eq. (1) is linear and homogeneous, in that it linvolves the current and its time derivatives or integrals in a linear fashion, and only a steady-state ac of frequency $\omega$ is produced by steady-state ac driving voltage. Fo: a nonlinear system, the situation is quite different.

In a nonlinear system, the equation relating the current to the applied voltage contains nonlinear expressions. These nonlinear expressions may contain the voltage, the current, and/or their time derivatives or integrals. For example, consider the generation of direct current by an ac voltage in a nonlinear circuit for the case where the instantaneous current and voltage are related as follows:

$$
I(t)=f(V(t)),
$$

where $I=0$ if the driving voltage is identically zero for a passive dissipative system and where $f$ is some nonlinear function of the voltage. To obtain the current response for a small ac voltage, we expand $f(V)$ in a power series about $\mathrm{V}=0$. That is,

$$
I(t)=f^{\prime}(0) V+\frac{1}{2} f^{\prime \prime}(0) V^{2}+\cdots,
$$

where the prime denotes differentiation with respect to $\mathrm{V}$. For $\mathrm{V}=\hat{\mathrm{V}}_{\mathrm{sin}}(\omega t)$, the current is given by

$$
I(t)=f^{\prime}(0) \hat{V} \sin (\omega t)+\frac{1}{2} f^{\prime \prime}(0) \hat{V}^{2} \sin ^{2}(\omega t)+\ldots .
$$

Noting that $\sin ^{2}(\omega t) \equiv \frac{1}{2}(1-\cos (2 \omega t))$, we have

$$
I(t)=\frac{\hat{v}^{2}}{4} f^{\prime \prime}(0)+f^{\prime}(0) \hat{V} \sin (\omega t)-\frac{1}{4} f^{\prime \prime}(0) \hat{V}^{2} \cos (2 \omega t) \ldots
$$


Hence, the nonlinearity, $f^{\prime \prime}(0) \neq 0$, has allowed the sinusoldal voltage to generate a direct-current component

$$
I_{d c}=\frac{\hat{v}^{2}}{4} f^{\prime \prime}(0)
$$

and a second harmonic with an amplitude equal to the dc value. If higher order terms in the expansion are kept, higher hrrmonics and further contributions to the dc component are generated. If the higher terms are negligible, the first and second harmonic ripple factors are

$$
R_{\omega}=\hat{I}_{a c} /\left|I_{d c}\right|=(4 / \hat{V})\left|f^{\prime}(0)\right| /\left|f^{\prime \prime}(0)\right|
$$

and

$$
R_{2 \omega}=1
$$

The generation of the direct current from a nonlinear current-voltage curve is shown schematically in Fjg. 2(a) (the imperfect rectifier). For comparison, an $f$ ieal rectifler with a corstant series resistance, where $f=0$ for $V<0$ and $f$ Is linear for $V>0$, is shown in $\mathrm{Fig}$. $2(\mathrm{~b})$.
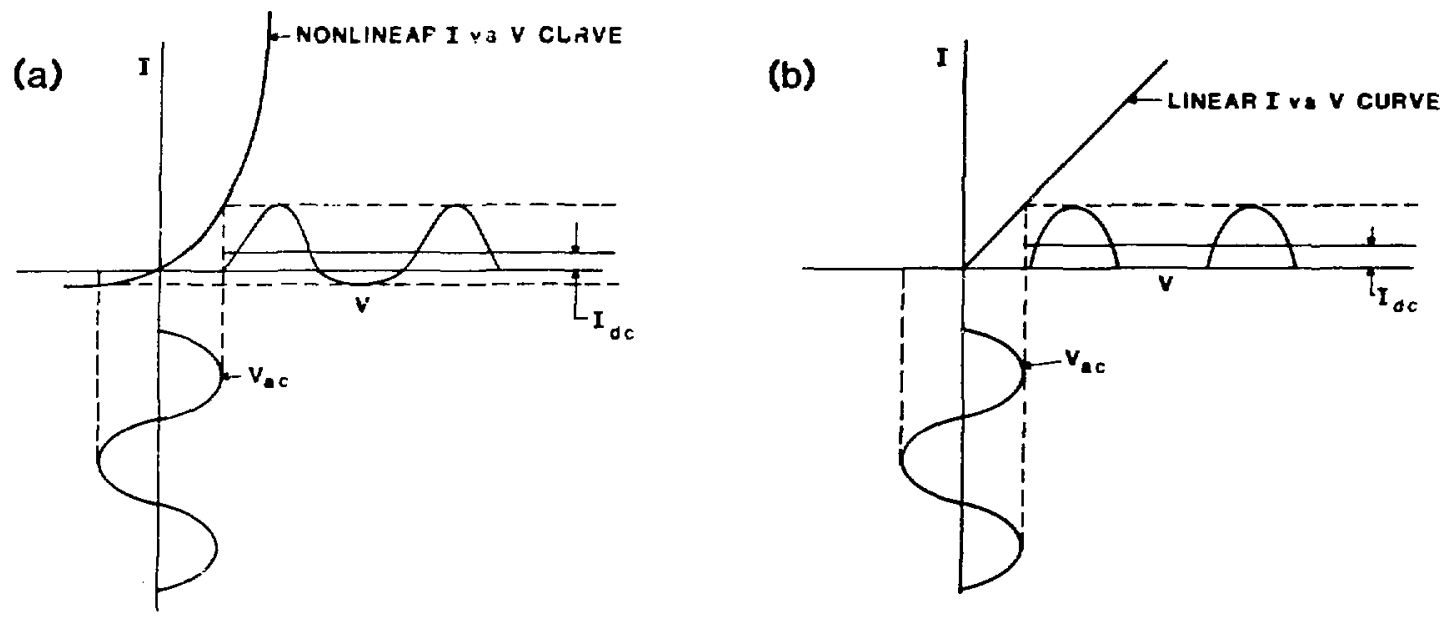

Fig. 2.

Nonlinear systems representing an (a) imperfect rectifier and (b) ideal rectifier. 


\section{B. The Two-Port System}

We now proceed in an analogous fashion to examine the production of a direct current from a single-frequency, sinusoldaliy driven two-port (twoterminal pair) system (Fig. 3). For a linear, passive two-port system in ac steady state, the relationship analogous to Eq. (1) is given by

$$
\begin{aligned}
& I_{1}(\omega)=Y_{11}(\omega) V_{1}(\omega)+Y_{12}(\omega) V_{2}(\omega) ; \\
& I_{2}(\omega)=Y_{21}(\omega) V_{1}(\omega)+Y_{22}(\omega) V_{2}(\omega),
\end{aligned}
$$

where the $\mathrm{Y}_{\mathrm{ij}}$ 's are elements of the complex admittanc matrix. As before, steady-state ac voltages can produce only steady-state ac currents with the same frequency.

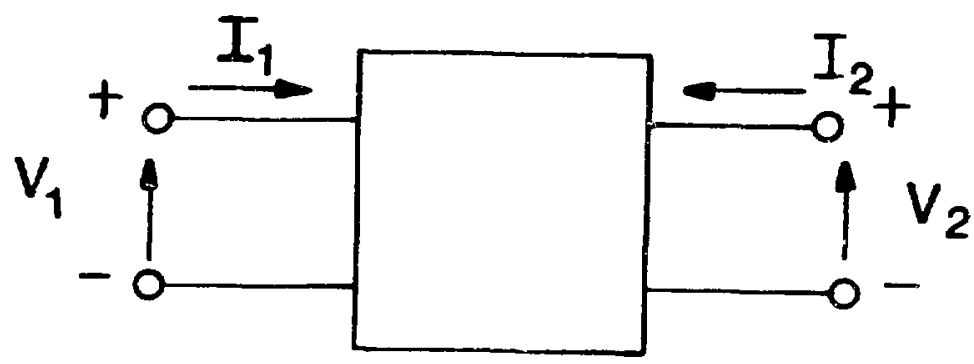

Fig. 3 .

Two-port system.

As an example of direct-current generation in a nonlinear two-port system, we assume a relationship analogous to $\mathrm{Eq} \cdot(2)$ :

$$
\begin{aligned}
& I_{1}(t)=F_{1}\left(V_{1}(t), V_{2}(t)\right), \\
& I_{2}(t)=F_{2}\left(V_{1}(t), V_{2}(t)\right),
\end{aligned}
$$

with $F_{1}(0,0)=-{ }_{2}(0,0)=0$. 
Expanding $I_{1}$ and $I_{2}$ in a double power series about $V_{1}$ and $V_{2}=0$ ylelds

$$
I_{n}=\sum_{i=1}^{2} a(n) v_{i}+\frac{1}{2} \sum_{i=1}^{2} \sum_{j=1}^{2} a\left\{v_{j}\right) v_{f} v_{j}+\cdots,
$$

where

$$
\begin{array}{ll}
a_{1}^{(n)} \equiv \partial F_{n} / \partial v_{1} & (n=1,2), \\
a_{j j}^{(n)} \equiv \partial^{2} F_{n} / \partial v_{i} \partial V_{j} & (1, j=1,2),
\end{array}
$$

evaluated at $v_{1}=v_{2}=0$.

For the applied voltages $v_{i}=\hat{v}_{1} \sin (\iota \cdot r)$ and $v_{2}=v_{2} \sin (\omega t+\delta)$, where $\hat{v}_{1}$ and $\hat{v}_{2}$ are positive,

$$
\begin{aligned}
I_{n}(t) & =a_{1}^{(n)} \hat{v}_{1} \sin (\omega t)+a \hat{V}^{(n)} \hat{v}_{2} \sin (\omega t+\delta) \\
& +\frac{1}{2}\left[a_{11}^{(n)} \hat{v}_{1}{ }^{2} \sin ^{2}(\omega t)+2 a_{12}^{(n)} \hat{v}_{1} \hat{v}_{2} \sin (\omega t) \sin (\omega t+\delta)\right. \\
& \left.+a f_{2}^{(n)} \hat{v}_{2}{ }^{2} \sin ^{2}(\omega t+\delta)\right]+\ldots,
\end{aligned}
$$

where $a_{12} \equiv a_{21}$ (interchangeability of partial differentiation). Expanding $\sin (\omega t+\delta)=\sin (\omega t) \cos \delta+\sin \delta \cos (\omega t)$ and then collecting terms, assuming terms higher than second order are negligible, yields

$$
I_{d c}(n)=\frac{1}{4}\left[a_{11}^{(n)} V_{1}^{2}+2 a_{12}^{(n)} \hat{v}_{1} \hat{v}_{2} \cos \delta+a \cdot(n) \hat{v}_{2}^{2}\right]
$$

For positive $a_{i j}{ }^{\prime}$, Eq. (16) Is maximized for $\cos \delta=1$. The first harmonic amplitude is

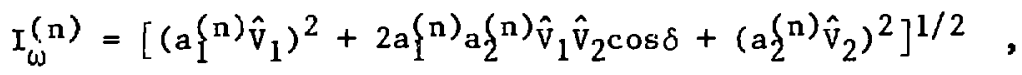


while the second hamonic amplitude is

$$
\begin{aligned}
I(\eta)= & \frac{1}{2}\left[\frac{1}{4}\left(a_{11}^{(n)} \hat{v}_{1}^{2}+2 a(n) \hat{v}_{1} \hat{v}_{2} \cos \delta+a(n) \hat{v}_{2}{ }^{2} \cos 2 \delta\right)^{2}\right. \\
& \left.+\left(a\{n) \hat{v}_{1} \hat{v}_{2} \ln \delta+\frac{1}{2} a_{2}(n) \hat{v}_{2}{ }^{2} \sin 2 \delta\right)^{2}\right]^{1 / 2} .
\end{aligned}
$$

We see that the two-port network introduces an extra degree of freedom aside from the network coefficients $a_{1}^{(n)}, a_{i j}^{(n)}$, namely, the phase $\delta$ between the ac driving voltages. For given values of the coefficients, the phase can be chosen to minimize the ripple. For $\delta=0$, assuming terms higher than the second order In Eq. (11) are negliglble, the ripple factor for the second harmonic becomes unity as it does for the one-port network.

\section{The More General Two-Port System}

A more general case, applicable to RFF modeling, is given by a pair of equations relating the voltages and currents:

$$
\begin{aligned}
& F_{1}\left(V_{1}, V_{2}, I_{1}, I_{2}, t, \ldots\right)=0, \\
& F_{2}\left(V_{1}, V_{2}, I_{1}, I_{2}, t, \ldots\right)=0,
\end{aligned}
$$

where the dots can represent derivatives and integrals of the voltages and currents. For the RFP model discussed in the next section, the variables are $v_{1}, V_{2}, I_{1}, I_{2}, \dot{I}_{2}, t$, and $s V_{1} d t$, where the subscript 1 denotes the poloidal $(\theta)$ varlables and 2 denotes toroldal $(\phi)$ vartables. We next generate the equations describiing the RFP as a nonlinear two-port system.

III. THE REVERSED FIELD PINCH AS A NONLINEAR CIRCUIT

The RFP is an axisymmetric toroldal contalnment device 4,5 in which plasma is confined by a pololdal magnetic fleld $\left(B_{\theta}\right)$, due to a toroldal plasma current, and a toroldal magnetic fleld $\left(B_{\phi}\right)$ generated by a pololdal plasma current and external windings. In contrast to the Tokamak, the RFP is characterized by a 
high-shear magnetic field configuration where the toroidal field is reversed on the plasma exterior with respect to its value on axis.

\section{A. The Taylor Model}

Taylor ${ }^{6}$ postulated, for the case of a plasma 1nside a perfectly conducting shell, that the RFP field configuration origiates from a process of field-1ine reconnection in which the plasma relaxes to a state of minimum field energy. In this model, the relaxed magnetic fields are elgenfunctions of the force-free equation

$$
\nabla \times \underset{\sim}{\mathrm{B}}=\lambda \underline{\sim},
$$

where $\lambda$ is a constant. The symmetric solution to Eq. (18), in the cylindrical approximation, ${ }^{*}$ is given by the Bessel function field profiles $B_{\phi}(r)=B_{o} J_{o}(\lambda r)$, and $B_{\theta}(r)=B_{0} J_{1}(\lambda r)$, where $B_{0}$ denotes the toroidal field on axis and $r$ defines the minor radial coordinate.

The relaxed states are independent of initial conditions and can be described by the dimensionless parameters $i$ and $\theta$;

$$
\begin{aligned}
& F=\text { Fleld Reversal Parameter }=\frac{B_{\phi}(a)}{\left\langle B_{\phi}\right\rangle}=\frac{B_{\phi}(a)}{\left(2 / a^{2}\right) \int_{0}^{a} B_{\phi}(r) r d r} \equiv \frac{L_{o} I_{\theta}}{\Phi} \\
& \theta=\text { Pinch Parameter }=\frac{B_{\theta}(a)}{\left\langle B_{\phi}\right\rangle}=-\frac{B_{\theta}(a)}{\left(2 / a^{2}\right) \int_{0}^{a} B_{\phi}(r) r d r} \equiv \frac{L_{o^{2} \phi}}{\varepsilon \Phi} \text {, }
\end{aligned}
$$

where

\footnotetext{
* We shall use the cylindrical approximation to torus throughout this paper. The cylindrical coordinates will be dencied by $r, \theta$, $\phi$ instead of the more common $r$, $\theta, z$ in order to keep a closer 1dentification with a torus (major circumference $2 \pi R)$. The polofdal and toroldal coordinates are identified with $\theta$ and $\phi$ respectively. Toroidal correct neglected.
} 


$$
\begin{aligned}
& L_{0}=\text { Vacuum Toroidal Inductance }=\frac{\mu_{0} a^{2}}{2 R}, \\
& \varepsilon=\text { Inverse Toroldal Aspect Ratio }=a / R, \\
& \Phi=\text { Toroidal Flux }=\int_{0}^{a} B_{\phi}(r) 2 \pi r d r, \text { and } \\
& \Delta \text { denotes a cross-section average. }
\end{aligned}
$$

The symbol a denotes the conducting wall radius; $R$ is the major radius of the torus; and $I_{\theta}, I_{\phi}$ represent the poloidal current in the conducting wall and : he plasma current, respectively. The locus of relaxed states accessible to the RFP forms a curve in $\mathrm{F}-\theta$ space as shown in F1g. 4.

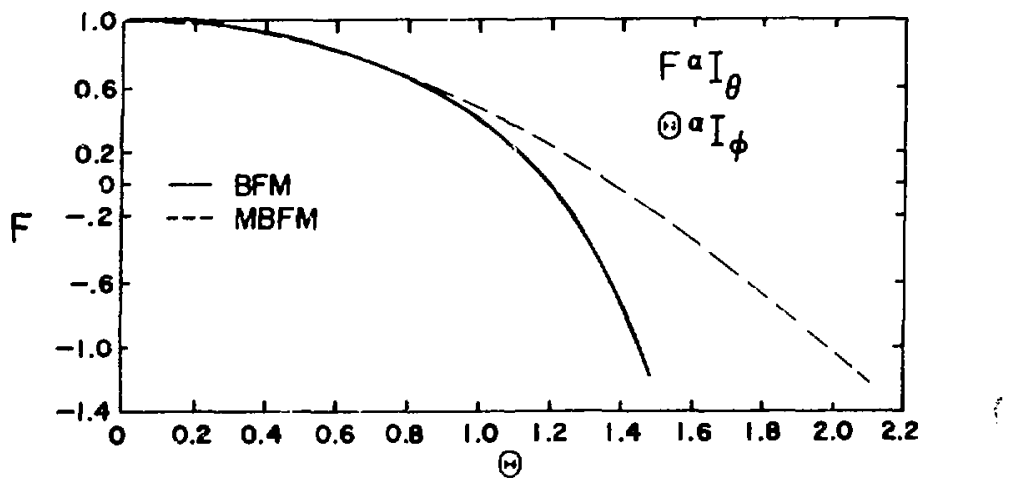

Fig. 4 .

The F- $\Theta$ curve. Here, BFM refers to the locus of relaxed states assuming Bessel function fleld profiles (Taylor curve).

The symmetric Bessel function fleld profiles may be expressed in terms of $\theta$ as $B_{\phi}=B_{0} J_{0}(2 \Theta r / a)$ and $B_{\theta}=B_{0} J_{1}(2 \Theta r / a)$. Taylor found that these field profiles represent minimum field energy states for $\lambda a<j .1$ (1.e., the first

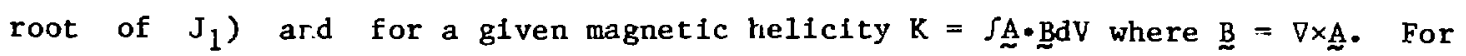
higher values of $\lambda a$, the lowest energy states are no longer axisymmetric.

\section{B. Derivation of the Nonlinear System Equation:}

Present RFP experiments differ from Taylor's model in that a perfectly conducting wall is not used. Instead the experiments usually employ a double wall consisting of a thin resistive vacuum liner surrounded by a thick, highly conducting shell. The shell contains gaps to allow flux to enter the plasma 
region during startup. In addition, the experimental RFP can be driven by voltages applied to poloidal and toroldal field windings located on the conducting she11. Thus, we are interested in extending Taylor's concept considerably beyond the simple relaxation model of a plasma inside a perfectly conducting, flux-conserving shell. We will therefore adopt the rollowing premises related to the idealized Taylor model:

(1) After the initial discharge formation, the plasma evolves to a relaxed state in a time that is short compared with that cf changes imposed by the externai driving circuits. The relaxed states are assumed to be cylindrically symmetric.

(2) There exists a unique locus in $F-\theta$ space on which the relaxed states 11 . Tt $a$ form of the field distributions depends only on the parameter $\theta$ and the magnitude of the fields scale with the instantaneous value of the toroldal flux.

(3) The relevant conducting wall radil $\underline{a}$ and $R$ are assumed to be those of the vacuum liner.

Using these premises, we may write the magnetic fieid components in the following functional form:

$$
B_{\phi}=B_{o}(\Phi) f_{\phi}(\theta, r)
$$

and

$$
B_{\theta}=B_{o}(\Phi) f_{\theta}(\theta, r),
$$

wh e $\theta$ and $\Phi$ are defined by Eqs. (20) and (23) as before.

From Eq. (23), the scale factor $B_{0}$ is related to the toroldal flux $\Phi(t)$ as follows:

$$
B_{0}=\Phi / \int_{0}^{a} f_{\phi}(\theta, r) 2 \pi r d r
$$

By Faraday's law, $\dot{\Phi}=-\int_{0}^{2 \pi} E_{\theta} a d \theta$ so that the toroidal flux is given by a time integral of the poloidal loop voltage. From Ampere's law, the toroidal plasma 
current $I_{\phi}$ and the single-turn-equivalent poloidal winding current $I_{\theta}$ are given by

$$
I_{\phi}=\left(2 \pi a / \mu_{0}\right) B_{\theta}(a) \equiv\left(2 \pi a / \mu_{0}\right) B_{o} f_{\theta}(\theta, a)
$$

and

$$
I_{\theta}=\left(2 \pi R / \mu_{0}\right) B_{\phi}(a) \equiv\left(2 \pi R / \mu_{0}\right) B_{o} f_{\phi}(\theta, a)
$$

The magnetic field energy in the plasma volume $V$ is

$$
W_{m}=\int_{V}\left(B^{2} / 2 \mu_{0}\right) d V=2 \pi R \int_{0}^{a} \frac{\left(B_{\phi}^{2}+B_{\theta}^{2}\right)}{2 \mu_{0}} 2 \pi r d r
$$

We now wish to obtain a pair of equations in the form of Eq. (17) that relate the currents $I_{\phi}$ and $I_{\theta}$ to the applied loop voltages $V_{\phi}$ and $V_{\theta}$. We will adopt the usual circuit theory sign conventions of Fig. 3; $1 . e .$, the product of a positive voltage and a positive current gives a power entering the system (the plasma volume). Thus the power entering the plasma volume will be $v_{\phi} I_{\phi}+v_{\theta} I_{\theta}$. This convention requires $v_{\phi}=\oint \mathrm{E}_{\phi} \mathrm{R} d \phi$ and $\mathrm{v}_{\theta}=-\oint \mathrm{E}_{\theta} \mathrm{a}$ d $\theta$ when the Iine integrals are evaluated by the right-hand rule.

The electroriagnetic power entering the system is given by Poynting's theorem

$$
\underset{S}{\int}(\underset{\sim}{E} \times \underset{\sim}{\mathrm{H}}) \cdot \underset{\sim}{\mathrm{n}} \mathrm{d} S=d W_{\mathrm{m}} / \mathrm{dt}+\int_{\sim}^{\mathrm{J}} \cdot \underset{\sim}{\mathrm{E}} \mathrm{dV}
$$

where $\hat{n}$ is the inward normal to the bounding surface element $d S, J$ is the plasma current density, and $w_{m}$ is given by Eq. (29). Defining an "effective plasma resistance" as

$$
R_{p} \equiv \underset{V}{J} \cdot \underset{\sim}{E} d V / I_{\phi}^{2}
$$

we can write the energy balance of $\mathrm{Eq} \cdot(30)$ as 


$$
v_{\phi} I_{\phi}+V_{\theta} I_{\theta}=d W_{r n} / d t+I_{\phi}^{2} R_{p}
$$

We may now express $W_{m}$ in terms of $I_{\phi}$ and $\theta$. This is most simply done by defining a function $L_{p}(\theta)$ called the "effective plasma inductance" such that

$$
W_{m}=\frac{1}{2} L_{p}(\theta) I_{\phi}^{2},
$$

where from Eqs. (24), (25), (27), and (29)

$$
L_{p}(\theta)=\frac{\mu_{o} R}{a^{2} f_{\theta}^{2}(\theta, a)} \int_{o}^{a}\left[f_{\phi}^{2}(\theta, r)+f_{\theta}^{2}(\theta, r)\right] r d r
$$

Although this expression for $\mathrm{L}_{\mathrm{p}}(\theta)$ is suitable for analyses, it becomes singular at $\theta=0$. This singularity gives rise to difficulties when rumerical simulations, starting at $I_{\phi}=0$, are performed. The difficulty basically results from the fact that the toroidal field energy is finite when $I_{\phi}=0$. At first it would seem that a natural solution to the problem would be to define $W_{m}$ $=\frac{1}{2} L_{\phi} I_{\phi}^{2}+\frac{1}{2} L_{\theta} I_{\theta}^{2}$ using two inductance parameters based separateiy on the toroidal and poloidal magnetic field energies. This makes $L_{\phi}$ finite for all $\theta$. However, for the RFP, $\mathrm{L}_{\theta}$ becomes singular at the $\theta$ value corresponding to the case where the toroidal field vanishes at $r=a$, since at that $\theta$ value, $I_{\theta}$ is zero but the toroidal magnetic field energy is not. To avoid all singular behavior, one may add a well-behaved function to $\mathrm{Eq}$. (33) that reduces to the toruidal field energy at $\theta=0$. One chofce of such a function gives

$$
\mathrm{W}_{\mathrm{m}}=\frac{1}{2} \mathrm{~L}_{\mathrm{p}}(\theta) \mathrm{I}_{\phi}{ }^{2}+\frac{\Phi^{2}}{2 \mathrm{~L}_{\mathrm{o}}},
$$

corresponding to 


$$
L_{p}(\theta)=\frac{\mu_{o} R}{a^{2} f_{\theta}^{2}(\theta, a)}\left[\int_{0}^{a}\left[f_{\phi}^{2}(\theta, r)+f_{\theta}^{2}(\theta, r)\right] r d r-\frac{2}{a^{2}}\left[\int_{0}^{a} f_{\phi}(\theta, r) r d r\right]^{2}\right\} .
$$

Note that $L_{p}(\theta)$ is nonsingular if $f_{\phi}(0, r)$ is a constant (i.e., the vacuum field).*

We now proceed to obtain a pair of equations for the RFP in the form of Eq. (17). Using $L_{p}(\theta)$ defined from Eq. (35), Eq. (32) can be written as

$$
\mathrm{d} / \mathrm{dt}\left[\frac{1}{2} \mathrm{~L}_{\mathrm{p}} \mathrm{I}_{\phi}^{2}+\Phi^{2} /\left(2 \mathrm{~L}_{0}\right)\right]-\mathrm{v}_{\phi} \mathrm{I}_{\phi}-\mathrm{V}_{\theta} \mathrm{I}_{\theta}+\mathrm{I}_{\phi}^{2} \mathrm{R}_{\mathrm{p}}=0
$$

or

$$
L_{p}(\theta) I_{\phi} \dot{I}_{\phi}+\frac{1}{2} L_{p}^{\prime}(\theta) \dot{\theta} I_{\phi}^{2}+\Phi \dot{\Phi} / L_{o}-V_{\phi} I_{\phi}-v_{\theta} I_{U}+I_{\phi}^{2} R_{p}=0
$$

where dots represent time differentiation and primes denote differentiation with respect to $\theta$. Differentiating Eq. (20) and using Faraday's law with the present sign convention $V_{\theta}=\dot{\Phi}$, one obtains

$$
\dot{\theta}=\frac{L_{o}}{\varepsilon \Phi}\left(\dot{I}_{\phi}-I_{\phi} V_{\theta} / \Phi\right)
$$

Substitution for $\dot{\theta}$ in Eq. (38) then gives

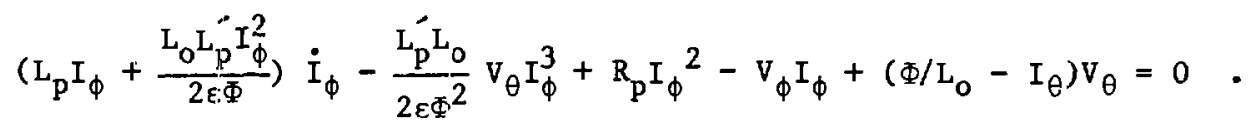

\footnotetext{
*The simplest, nonsingular $\mathrm{L}_{\mathrm{p}}(\theta)$ is obtained from Eq. (36) by setting $\theta \equiv 0$ in the last integral on the right. However, in this paper, we have chosen $L_{p}(\theta)$ as defined from Eq. (35) for continuity with previous work.7 [The definition of Eq. (35) was provided by A. A. Newton, Culham Laboratory, AbIngdon, England, 1981.] If Eq. (33) is used instead of Eq. (35) to define $L_{p}$, the effect is to
replace the factor (1-F) by $-F$ in Eq. (43).
} 
From the postulated $F-\theta$ curve we obtain $F=F(\theta)$, where in terms oí the dimensionless field distributions of Eqs. (24) and (25),

$$
F(\theta)=\frac{1}{2} a^{2} F_{\phi}(\theta, a) / \int_{0}^{a} f_{\phi}(\theta, r) r d r
$$

Using Eq. (19), $I_{\theta}$ may then be expressed as

$$
I_{\theta}=\left(\Phi / L_{0}\right) F(\theta)
$$

If we note that $\Phi=\int V_{\theta} d t$ and the definition of $\theta$, we see that the equation pair (40) and (42) is in the form of Eq. (17). We can also obtain a singie equation for $I_{\phi}$ by substituting $I_{\theta}$ from Eq. (42) into Eq. (40). Further elimination of $\Phi$ in favor of $\theta$ using $\mathrm{Eq}$. (20) yields

$$
I_{\phi} R_{p}-V_{\phi}+\left[L_{p}+(\theta / 2) L_{p}^{\prime}\right] \dot{I}_{\phi}+\left[\frac{1-F}{\varepsilon \theta}-\frac{\varepsilon \theta^{2} L_{p}^{\prime}}{2 L_{o}}\right] v_{\theta}=0
$$

Equation (43), although complex, illustrates the important nonlinear plasma-circuit properties. ne RFP. This nonlinear coupling between the toroidal current and the dri. voltages arises via the $F-\theta$ diagram. The nonlinear plasma circuit behavior described by Eqs. (42) and (43) forms the basis for the OFCD concept.

IV. SMALL-SIGNAL ANALYSIS OF THE DIRECT-CURRENT GENERATION IN THE RFP FROM OSCILLATING APPLIED VOLTAGES (F- $\theta$ PUMPING)

In the spirit of Sec. II, we now comment on small-signal periodic solutions of Eqs. (40) and (42) for assumed sinusoidal driving voltages of radian frequency $\omega$ as follows:

$$
\begin{aligned}
& v_{\theta}=\hat{v}_{\theta} \sin (\omega t) ; \\
& v_{\phi}=v_{\phi} \sin (\omega t) ; \tilde{v}_{\phi} \cos (\omega t) \equiv \hat{v}_{\phi} \sin (\omega t+\delta),
\end{aligned}
$$


where $\hat{v}_{\phi}=\left(v_{\phi}^{2}+\widetilde{v}_{\phi}^{2}\right)^{1 / 2}$ and $\delta=\tan ^{-1}\left(v_{\phi} / \widetilde{v}_{\phi}\right)$

We assume, in the smal! signal approximation, that variations of quantities about the operating point values of $e$ and $\Phi$, denoted by $\Theta_{0}$ and $\Phi_{0}$, are small. Expanding $L_{p}$ and $F$ in a Taylor series about $\theta_{o}$ yields

$$
L_{p}(\theta)=L_{p}\left(\theta_{0}\right)+L_{p}^{\prime}\left(\theta_{0}\right)\left(\theta-\theta_{0}\right)+\ldots
$$

and

$$
F(\theta)=F\left(\theta_{0}\right)+F^{\prime}\left(\theta_{0}\right)\left(\theta-\theta_{0}\right)+\ldots
$$

Integrating Eq. (44) gives

$$
\Phi=\int \mathrm{v}_{\varepsilon} \mathrm{dt}=-\left(\hat{\mathrm{v}}_{\theta} / \omega\right) \cos (\omega t)+\Phi_{0}
$$

For a periodic solution, $I_{\phi}$ and $R_{p}$ may be expanded in Fourier series

$$
I_{\phi}(t)=I_{0}+\sum_{n=1}^{\infty}\left[\left(I_{n} \sin (n \omega t)+\tilde{I}_{n} \cos (n \omega t)\right]\right.
$$

and

$$
R_{P}(t)=R_{0}+\sum_{n=1}^{\infty}\left[R_{n} \sin (n \omega t)+\tilde{R}_{n} \cos (n \omega t)\right] .
$$

The quantity $I_{0}$ is the dc component of the toroidal plasma current of primary interest for current drive. The corresponding expressions for $\theta$ and $\theta^{-1}$ can be obtained using Eqs. (20), (48), and (49), expanding about the operating point $\theta_{0}=L_{0} I_{0} / \varepsilon \phi_{0}$. Substituting these expressions into Eq. (43) and equating the coefficients of $\sin (n \omega t)$ and $\cos (n \omega t)$ for each $n$ value to zero, one obtains a system of equations. The voltages are regarded as prescribed, and the $R_{p}(t)$, $L_{p}(\theta), \quad F(\theta)$ functions and geometry constants $L_{o}$ and $\varepsilon$ are spectfic for a given RFP experiment. The desired quantities to be obtained are the corresponding direct current $I_{0}$ and the ac current ripple for each harmonic described by $I_{n}$ and $\tilde{I}_{n}$ for $n>0$. 
In general, the system of simultaneous equations contains poiynomials in the unknowns $I_{n}$ and $\tilde{I}_{n}$ whose coefficients are real, rational algebraic functions of the driving ac voltage coefficients $\hat{v}_{\theta}, v_{\phi}, \tilde{v}_{\phi}$; the frequency $\omega$; the values of the $F$ and $L_{p}$ functions and their first derivatives at the operating point $\theta_{0}$; the effective resistance parameters $R_{0}, R_{n}, \tilde{R}_{n}$; and the geometry parameters $L_{0}$, $\varepsilon$; and $\exists_{0}$. The degree and complexity of these equations depend strongly on the truncation and the ordering of small quantities that are used. The ordering of small quantities involves the unknowns and must be checked for self-consistency for the parameter values of interest after solutions to the system of equations are obtained. We shall reserve the parameter study of the small-signal solutions for a separate paper and proceed to a direct computational study of $F-\Theta$ pumping making use of Eqs. (42) and (43). However, it is instructive to first explore an alternate description of $F-\Theta$ pumping using magnetic helicity conservation.

V. F- $\Theta$ PUMPING AND MAGNETIC HELICITY CONSERVATION

The preceding description of $F-\Theta$ pumping as a consequence of the nonlinear behavior of RFP discharges is based cn a model of electromagnetic power balance (Poynting's theorem). An alternate description of the $F-\theta$ pumping concept can be formulated based on a model of magnetic helicity balance. ${ }^{2}$ Magnetic helicity in the RFP can be interpreted as the "knottedness" of magnetic field lines within the toroidal vacuum vessel excluding the coupling of plasma fields with externally linked fields. 8,9 That is,

$$
K=\int_{v}^{A} \underset{\sim}{\operatorname{Br}} \mathrm{d} \mathbf{v}-\Phi \Psi
$$

where $\underset{\sim}{A}$ is the vector potential such that $\underset{\sim}{B}=\nabla \times \underset{\sim}{A}$, $\Phi$ denotes the toroidal flux [(Eq. (23)], and $\Psi$ is defined as the cxternal pololdal flux threading the centrai hole of the torus (also called the magnetizing flux).

In an RFP with a conducting shell or equivalent equilibrium-stabilizing system, the component of $\underset{\sim}{B}$ normal to the shell surface is small and the time derivative of $\mathrm{Eq}$. (51) becomes $2,10,11$ 


$$
\frac{\mathrm{dK}}{\mathrm{dt}}=2 \Phi V_{\phi}-\underset{V^{\sim}}{2 \int \mathrm{B} d V}
$$

where $V_{\phi}$ denotes the toroidal loop voltage.

In Eq. (52), the term $2 \Phi V_{\phi}$ represents the magnetic helicity input per unit time while the Lerm $2 \int_{\sim}^{E} \bullet \underset{\sim}{B} d V$ denotes the total helicity dissipation per unit time within the plasma. In steady state, $\frac{d}{d t}\langle K\rangle=0$, which implies

$$
\left\langle\Phi \mathrm{V}_{\phi}\right\rangle=\left\langle\int_{\sim}^{\mathrm{E}} \cdot \underset{\sim}{\mathrm{Bd}} \mathrm{V}\right\rangle
$$

where $〈$ denotes a time average. For normal inductive drive of an RFP experiment, where the toroidal flux and plasma current are helá constant, helicity balance [(Eq. (53)] is maintalned by a dc toroldal voltage $\left(\mathrm{V}_{\phi}\right)$, However, because $v_{\phi}=-\dot{\Psi}_{m}$, Inductive drive does not yield a true steady-state current-drive solution.

Bevir and Gray ${ }^{2}$ first suggested that heliclty balance and hence the plasma current could be maintained in a mean steady state by simultaneously modulating both the toroldal voltage and toroldal flux. For example, consider the toroidal and pololdal sinusoldal driving voltages $\mathrm{v}_{\theta}=\hat{\mathrm{v}}_{\theta} \sin (\omega t)$ and $\mathrm{v}_{\phi}=\hat{\mathrm{v}}_{\phi} \sin (\omega t+\delta)$ from Eqs. (44) and (45). From Eq. (48), $\Phi=\int v_{\theta} d t=-\left(\hat{V}_{\theta} / \omega\right) \cos (\omega t)+\Phi_{0}$, and thus evaluating the left-hand side of Eq. (53) yields

$$
\begin{aligned}
\left\langle\phi \mathrm{V}_{\phi}\right\rangle & =-\frac{\hat{\mathrm{V}}_{\theta} \hat{\mathrm{V}}_{\phi}}{\omega}\langle\cos (\omega t) \sin (\omega t+\delta)\rangle \\
& =-\frac{\hat{\mathrm{V}}_{\theta} \hat{\mathrm{V}}_{\phi}}{2 \omega} \sin \delta, \\
& =\frac{\hat{\mathrm{V}}_{\theta} \hat{\mathrm{V}}_{\phi}}{2 \omega} \text { for } \delta=-\pi / 2 .
\end{aligned}
$$

Thus the applied ac voltages are most effective in supplying the helicity when $v_{\phi}$ lags $V_{\theta}$ by $y 0^{\circ}$. If the dissipacion term $\left.\left\langle\int \underset{\sim}{E} \cdot \underset{\sim}{B d}\right\rangle\right\rangle$ is not adversely affected 
by the sinusoidal driving voltages, then Eq. (53) demonetrates steady-state helicity balance because both $\left\langle V_{\phi}\right\rangle$ and $\left\langle V_{\theta}\right\rangle$ are zero. This, of course, implies from time averaging Faraday's Law that $\langle\Phi\rangle$ and $\left\langle\Psi_{\mathrm{m}}\right\rangle$ are constant in time.

The conjecture that steady-state helicity balance implies steady-state current drive relies on the nonlinear coupling as discussed earlier. The same set of assumptions previously invoked in the power-balance model (i.e., the pinch remains in a quasi-ralaxed state during the voltage modulations and there exists a unique locus in $F-\theta$ space on which the relaxed states lie) implies that the sinusoldal driving frequency must remain below the inverse characteristic relaxation time of the pinch. Also, it is crucial that the driving terms do not adversely affect the time-averaged helicity dissipation. 11 the validity of these assumptions must ultimately be tested by experiment.

\section{ZERO-DIMENSIONAL SIMULATIONS OF F- $\Theta$ PUMPING}

As stated earlier, the Taylor model and assumptions do not totally correspond to actual RFP experiments. In general, the measured F- $\theta$ curve lies above the corresponding Taylor prediction (see Fig. 4). H: cause our aim is to demonstrate the dc drive properties for parameters believed to be relevant in RFP experiments, we must make specific choices for the $F(\theta), L_{p}(\theta)$, and $R_{p}(t)$ functions.

The furction $F(\theta)$ can be obtained experimentally since $F$ and $\theta$ are easily measured by external electrical diagnostics. However the $L_{p}$ and $R_{p}$ quantities involve internal fleld measurements that entail inserting magnetic field probes into hot plasmas. For the high current and long pulse duration in the operating ranges of interest, the insertion of marerial probes seriously perturbs the plasma behavior. In addition, there is a risk of probe breakage and serious contamination of the vacuum chamber. In the absence of internal field mesurements, $L_{p}$ and $R_{p}$ are determined from a specific model for the field distributions of Eqs. (24) and (25). The objective is to use a model that is close to the Taylor-Bessel functions, but modifled to give agreement with the experimental F- $\Theta$ diagram.7,11 Using the approximation that the cross-field current flow is small in low beta plasmas, we use the modified force-free equation

$$
\nabla \times \underline{B}=\lambda(r) \underline{\mathrm{B}}
$$


where $\lambda$ is no longer a constant (as in Taylor theory) but is a function of minor radius and specifies the current profile. Furthermore, we choose a $\lambda$ profile that drops to zero at the wall* (see Fig. 5). The resulting field configuration is called the modifled Bessel function model (MBFM) (not to be identified with the modified Bessel functions of the mathematics 1tterature). Figure 6 shows an example of the MBFM field and current density profiles. The corresponding MBFM $\mathrm{F}-\theta$ curve is shown in Fig. 4. Details of this model are given in Refs. 7 and 11.

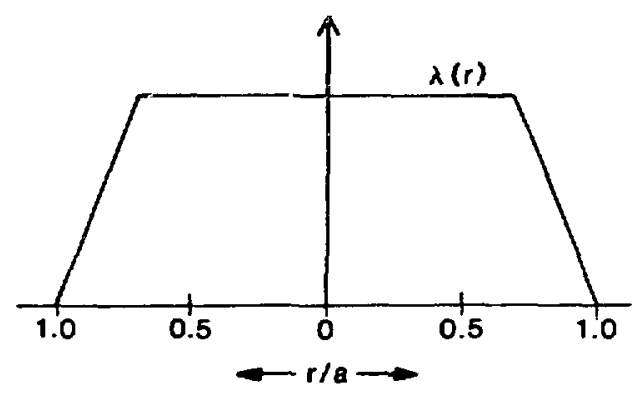

Fig. 5.

Spatial profile of $\lambda(r)$ used in generating the fields of Fig. 6 .

(a)

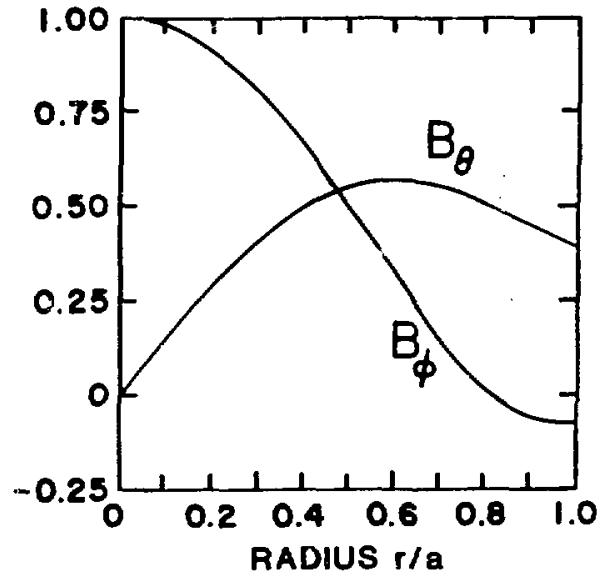

(b)

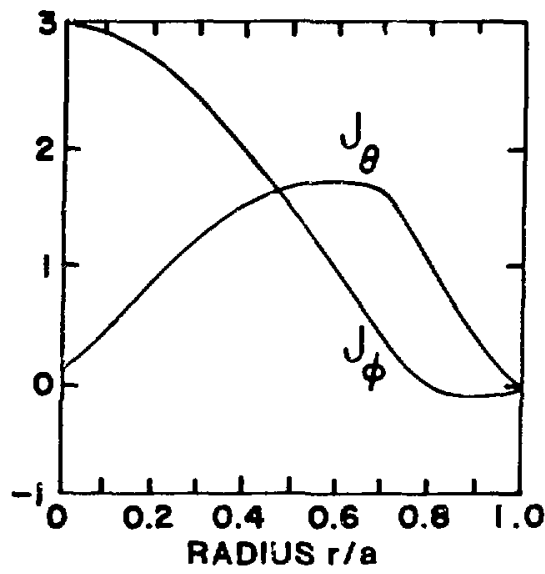

Fig. 6 .

Toroidal and poloidal spatial profiles from the MBFM for (a) magnetic fleld and (b) current density. ॠ This model was proposed by A. A. Newton, Culham Laboratory, Abingdon, England,
1981 . 
With these profiles, which have been parameterized to give a good fit to a given experimental $F-\theta$ diagram and voltage and current waveforms, $I_{p}(\theta)$ is computed from Eq. (36). $R_{p}$ may then be calculated from Eq. (32).7

\section{A. Simulations of $F-\theta$ Pumping on $2 T-40 \mathrm{M}$}

A conceptual diagram of an RFP is shown in Fig. 7. It consists of a metal vacuum vessel surrounded by a close-fitting conducting shell. The purpose of the shell is to provide the initial plasma equilibrium and stabilize the plasma against MHD instabilities. The toroidal and the poloidal magnetic fields are initlated and sustained by currents flowing in the field windings external with the shell. Currents flowing in the poloidal field windings also provide the vertical field necessary for plasma equilibrium on timescales that are long compared with the shell diffusion time.

\section{TOROIDAL AFP}

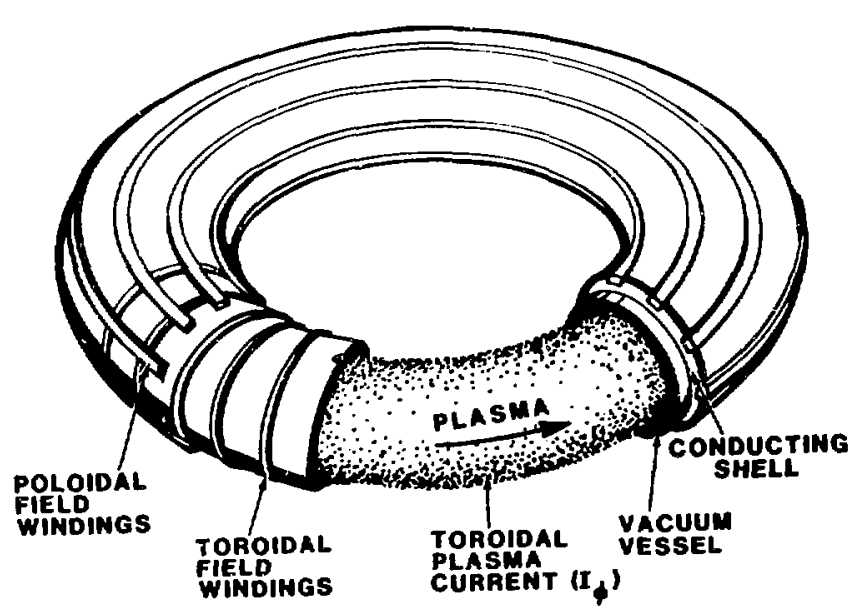

Fig. 7 .

Conceptual diagram of the RFP.

An equivalent electrical schematic of the ZT-40M RFP system is shown in FIg. 8. Both the poloidal and toroidal magnetic field circuits are driven by EMF sources that typically utilize capacitor banks. The schematic illustrates that the RFP may be treated as a two-port system 1 which $I_{\theta}$ and $V_{\theta}$ represent, respectively, the single-turn equivalent (s.t.e.) pololdal winding current and voltage. $V_{\phi}$ is the s.t.e. toroidal winding voltage and $I_{\phi}$ is the plasma current. The coupling of the RFP plasma to ths esternal circult equations is 


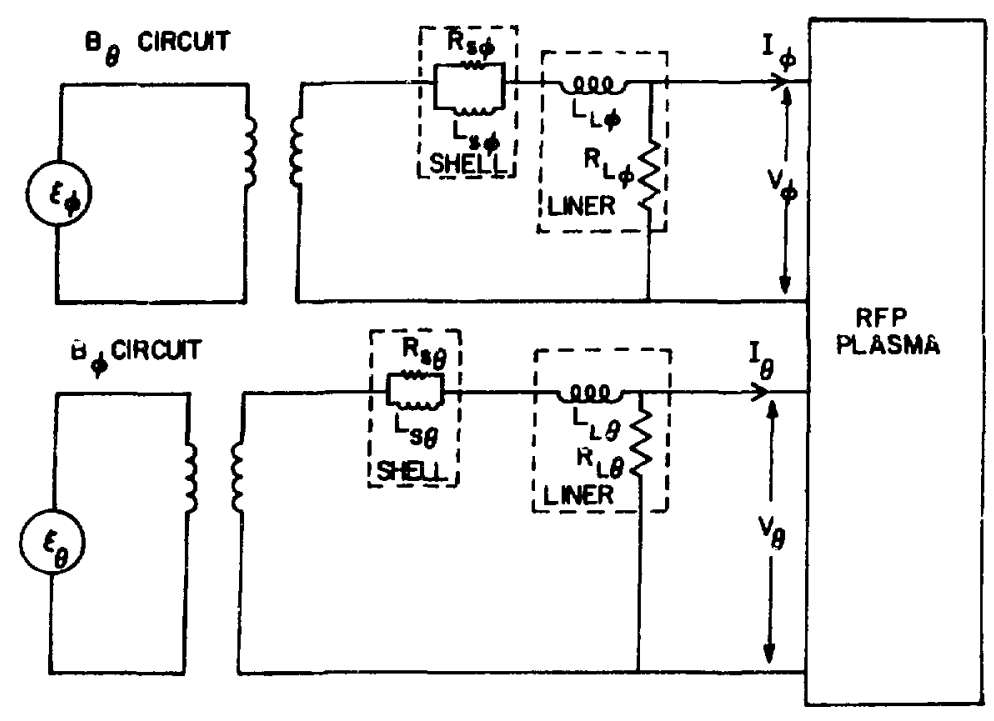

Fig. 8 .

Conceptual schematic of the RFP.

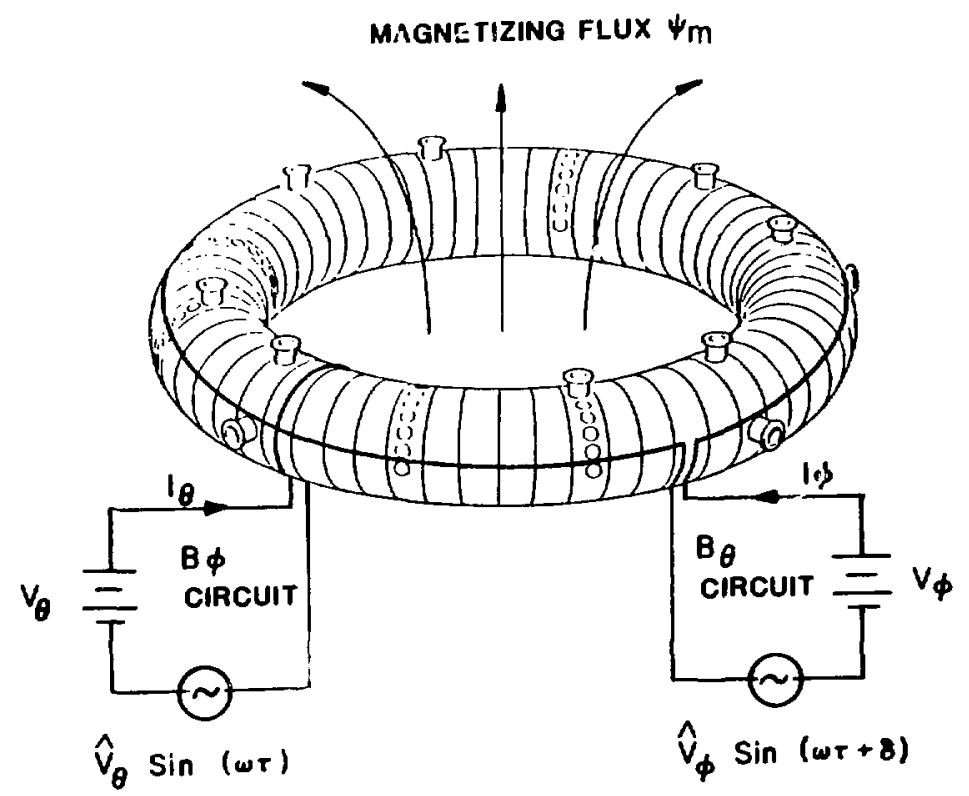

F1g. 9.

oscillating fleld current-drive schematic. 
described by Eqs. (42) and (43). Figure 8 thas forms the conceptual basis of a zero-dimensional computational code that, to date, has successfully simulated the electrical behavior of RFP discharges ${ }^{7}$. The remainder of this section discusses the application of this code to evaluating the OFCD concept.

Figure 9 illustrates a conceptual schematic of an OFCD system. The system consists of de supplies to provide pinch initiation and startup in series with the ac drivers. The ac drivers are essentially phase-locked variable amplitude audio oscillators. For steady-state operation, the $B_{\phi}$ de supply provides a negative toroidal field bias, and the $B_{\theta} d c$ supply drives only the external circuit ohmic drop in order to maintain plasma equilibrium. The combined and properly phased $V_{\theta}$ and $v_{\phi}$ oscillators drive the plasma current by means of the nonlinear $\mathrm{F}-\Theta$ coupling.

The $F-\Theta$ pumping concept was initially studied by simulating the effect of ac $V_{\phi}$ and $V_{\theta}$ sources on the Los Alamos ZT-40M experiment.12,13 In these simulations, the MBFM field profiles and concomitant $L_{p}(\theta)$ were chosen to give a good fit to an exper:-ntal ZT-40M F- $\Theta$ diagram (Fig. 4). The plasma resistance, $R_{p}$, was chosen to give the best fit to the experimental ZT-40M current and voltage waveforms when the MBFM model is used in computer simulations.7 Figure 10 shows the simulation results for a 200-kA discharge modulated at $2.5 \mathrm{kHz}$. Figures $10(\mathrm{a})$ and $10(\mathrm{~b})$ illustrate the effect of $F-\theta$ pumping on sustalning the plasma current and mean toroldal fleld waveforms. The etfect of the ac drive is apparent. Figure $10(\mathrm{c})$ shows the time history of the magnetizing flux $\Psi_{\mathrm{m}}$ that threads the central hole of the torus. Note that the time-averaged magnetızing flux remains constant during current drive as is required for steady-state operation.

For a given RFP, current drive by $F-\Theta$ pumping is basically controlled by five parameters: the amplitudes, $\hat{v}_{\theta}$ and $\hat{V}_{\phi}$, of the ac modulating voltages; the phasing $\delta$ between the voltages; the operating point $\theta_{0}$; and the driving frequency $\omega$. The simulation resulis illustrated in Fig. 10 were optimized in order to balance the ac power requirements in the toroldal and pololdal field circults. This was accomplished by first adjusting the phase $\delta$ between $V_{\phi}$ and $v_{\theta}$ to maximize the current-drive effect. Optimum performance required $v_{\phi}$ to lag $v_{\theta}$ by $90^{\circ}$ as shown in Fig. $10(\mathrm{~d})$, which is consistent with the predictions of the magnetic helicity model given by $\mathrm{Eq} .(54)$. The drivini frequency was selected to be much larger than an inverse discharge iffetime and smaller than an inverse plasma relaxation time. Finally, the poloidal ac driving voltage 


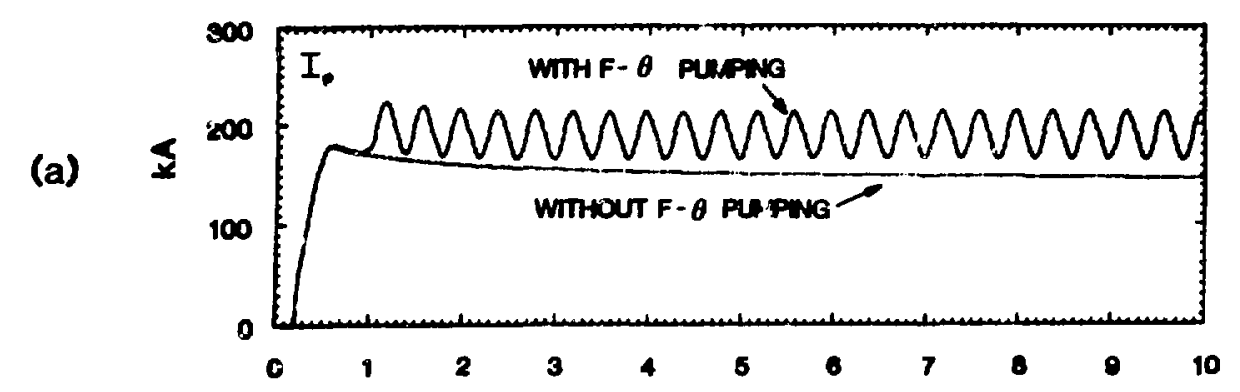

TME ( $m s$ )

(b)

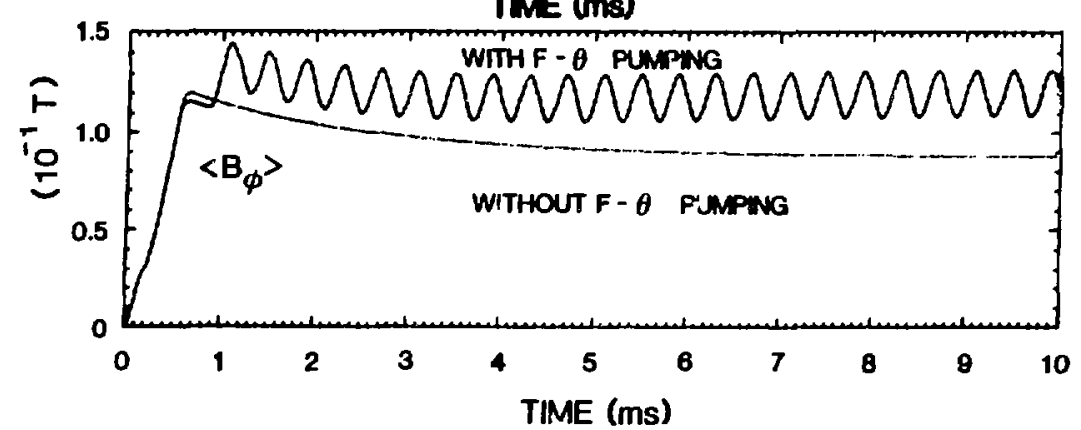

(c)

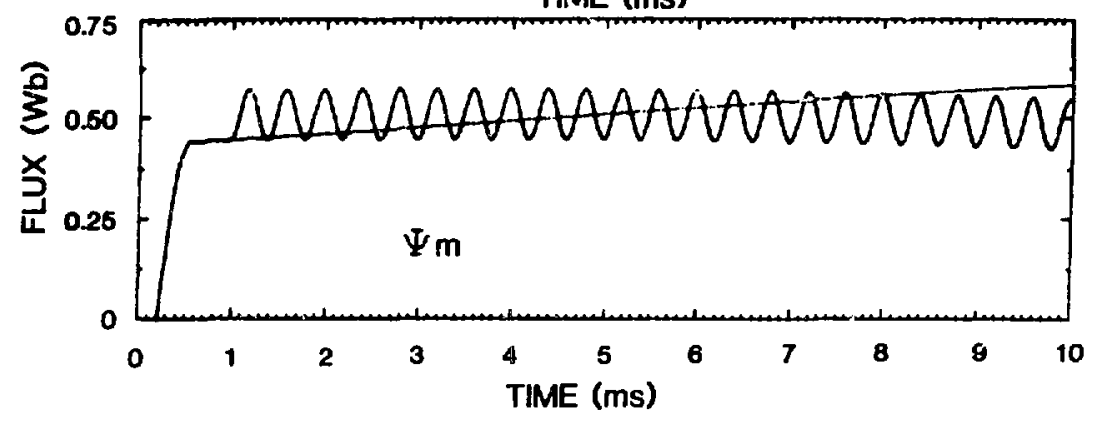

(d)

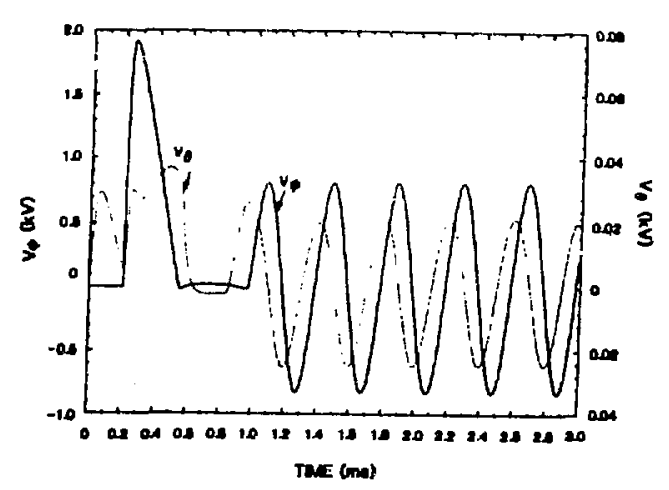

Fig. 10.

Computational simulations of F- $\theta$ pumping on ZT-40M: (a) plasma current with and without $F-\theta$ pumping, (b) mean toroidal field with and without $F-\theta$ pumping, and (c) simulated waveform for the magnetizing flux ( $\left.\Psi_{m}\right)$ with and without $F-\theta$ pumping. Here, $\Psi_{m}$ denotes the magnetic flux threading the central hole of the torus. (d) Applied voltages $v_{\theta}$ and $v_{\phi}$ valuated at the plasma. 
$\left(V_{\theta}\right)$ was maximized subject to the constraint that the toroldal field at the wall remain reversed during modulation.

The simulation code used 0 -btain the results in $F i_{c} \cdot 10$ makes seviral assumptions regarding plasma behavior $i_{\text {, }}$ the RFP. These assumptions are

1) The modulation does not significantly degrade overall pinch performance. In particular, no overt deleterious effects are observed with respect to confinement, equilibrium, plasma-wall interactions, resistivity, etc.

2) During modulation, the plasma remains in a quasi-relaxed state. That is, the plasma's dynamic response to modulation remains along a well-defined trajectory in F- $\Theta$ phase space. This requires the plasma's relaxation time to be much shorter than one modilation period, which sets an upper limit on the driving frequency.

In order to test these assumptions, an experimental series was run on ZT-40M that entailed modulating the toroldal and poloidal field circuits during 120-kA discharges over the frequency range from $750 \mathrm{~Hz}$ to $1.4 \mathrm{kHz} .14$ No attempt was made during this exercise to drive current by $F-\Theta$ pumping, because simultaneous modulation of both field circuits required significantly moie power and phase control accuracy between the respective drivers than was avallable.

overall pinch behavior during modulation was monitored by a standard set of plasma diagnostics. Plasma parameters such as electron density, resistivity, Impurity content, and equilibrium position showed no substantial departures from normal 120-kA operation as long as the toroidal magnetic field reversal was adequately maintajned. Of particular interest were the data taken from a multichannel deuteriun-alpha $\left(D_{\alpha}\right)$ monitoring system that measures plasma-wa11 interactions. The $D_{\alpha}$ monitors did not show any sianificant increase in plasmawall interactions during modulation, even during the temporal periods when the Poynting $f l u x$ was outward. 14

As previously note', the magnetic field coupling in a RFP is defined by ine discharge's trajectory in $F-\Theta$ phase space. Figure 11 illustrates typical discharge behavior during one experimental modulation period. The bold curve represents the $F-\Theta$ trajectory predicted by the modified Bessel function model (MBFM) and used by the simulation. For the case where the toroidal voltage $\left(V_{\phi}\right)$ is modulated, the operating point of the discharge moves clockwise in an elliptical path where one elliptical revolution corresponds to one modulation period. Simllarly, when the poloidal voltage $\left(v_{\theta}\right)$ is modulated, the operating point moves counterclockwise around the ellipse. The major axis of the ellipse 


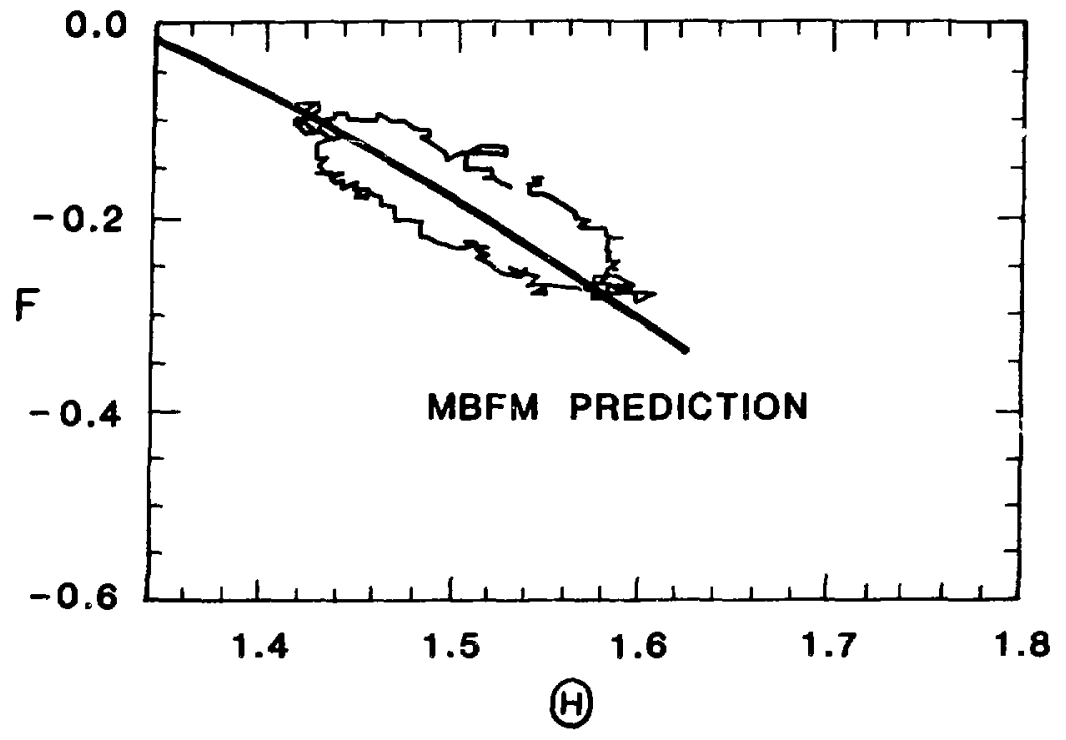

Fig. 11 .

An experimental F- $\theta$ trajectory for one modulation period during $1-\mathrm{kHz}$ wodulation. The bold curve shows the trajectory predicted by the modifled Bessel function model.

approximately corresponds to the predicted MBFM F- $\Theta$ trajectory for this run's conditions, and its length is dependent on the modulation amplitude of the voltage driver. The minor axls of the ellipse, and the orientation with which the operating point traverses the elliptical path, implies a 100- $\mu$ s time delay in

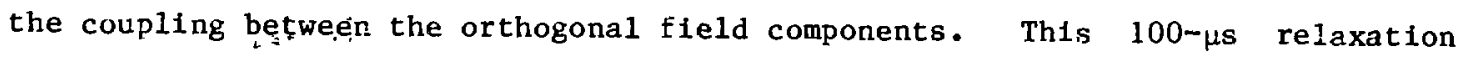
time is consistent with resistive tearing mode time scales for $2 \mathrm{~T}-40 \mathrm{M}$ and is much less than one modulation period.

The effect of a finite relaxation time on $F-\theta$ pumping was checked by incorporating a coupling delay in the current-drive simulations.* Figure 12(a)

\footnotetext{
* This was done by replacing $\theta$ in Eqs. (42) and (43) by the value obtalned by simultaneousiy integrating $\dot{\theta}=\left(L_{o} I_{\phi} / \varepsilon \Phi-\theta\right) / \tau_{d}$, where $\tau_{d}$ is a constant delay time parameter, instead of using Eq. (20).
} 
(a)

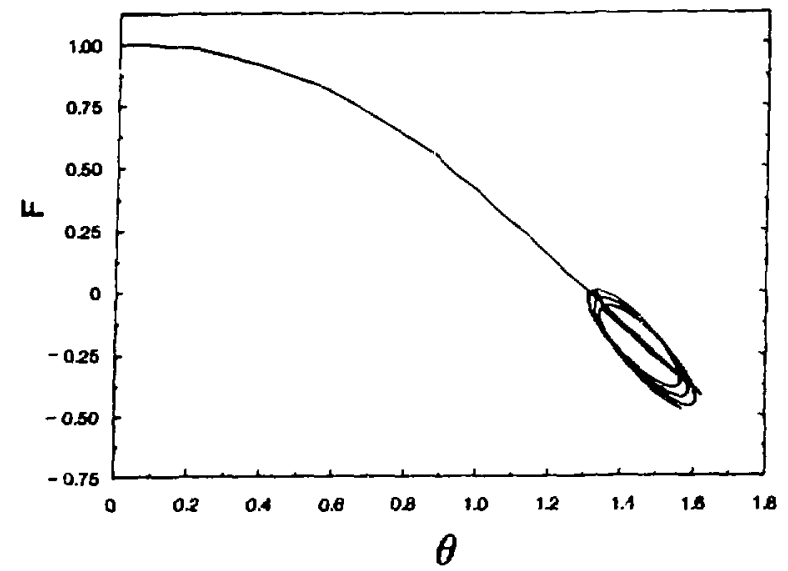

(b)
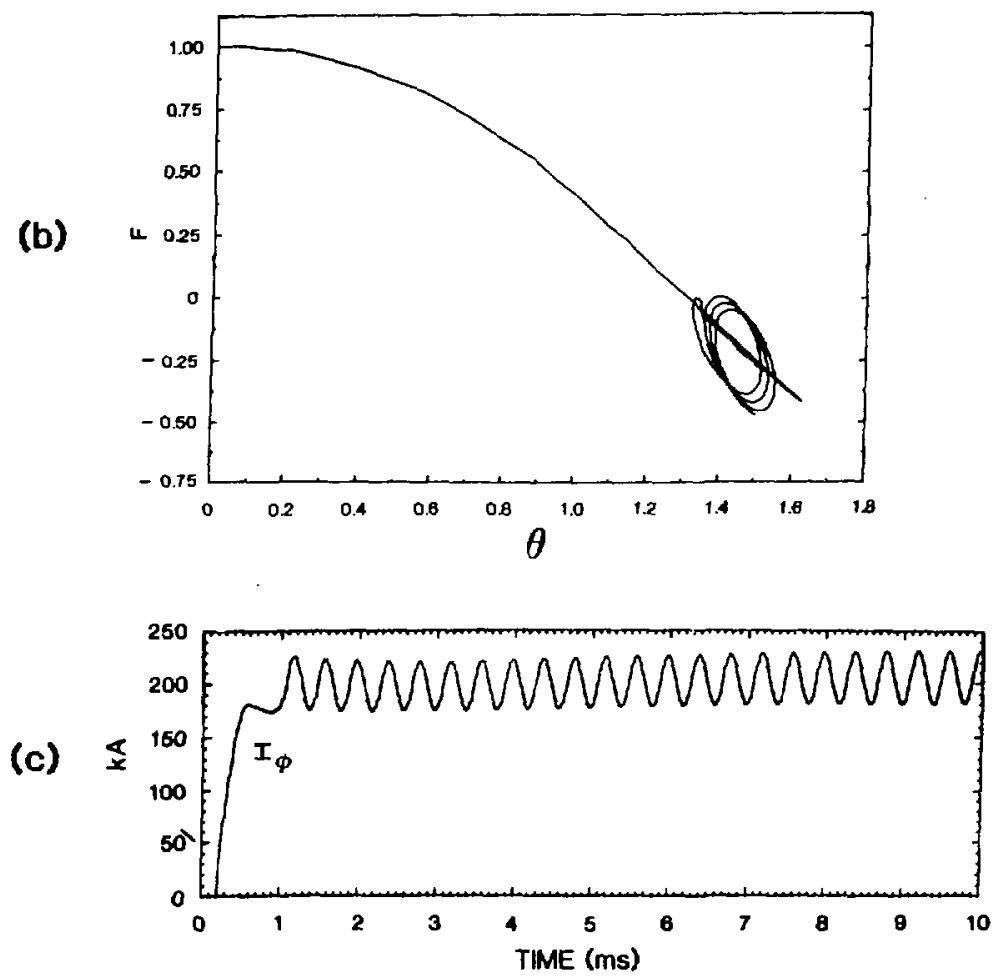

$$
\text { Fig. } 12 \text {. }
$$

(a) An $F-\theta$ trajectory for a simulated 40- $\mu$ s delay due to a finite relaxation time showing several modulation periods. This temporal delay corresponds to the same 0.6-rad phass daiay experimentally deduced from Fig. 11. (b) An $F-\theta$ trajectory for a $: 00-\mu s$ delay. (c) Corresponding simulated plasma current waveform for a 100- $\mu$ s delay. 
shows the resulting F- $\theta$ diagram for a 40- $\mu s$ delay. This temporal de:ay corresponds to the same phase delay ( $0.6 \mathrm{rad})$ that was experimentally deduced from Fig. 11. Figures $12(\mathrm{~b})$ and (c) show the simulated F- $\theta$ trajectory and plasma current waveform assuming a 100- $\mu$ s delay. This amount of delay does not adversely effect che nondelayed simulation results illustrated in Fig. 10.

\section{B. The Reactor Relevance of F-O Pumping}

The relatively large modulation amplitudes $\left(\hat{\mathrm{I}}_{\phi} /\left\langle\mathrm{I}_{\phi}\right\rangle \sim 10 \%\right.$ ) and concomitant power requirements shown in Fig. 10 illustrate that $F-\theta$ pumping is difficult to achieve in a low-temperature (200- to 300-eV) RFP plasma. However, F- $\theta$ pumping shows excellent promise as a viable current-drive mechanism for the RFP reactor. Recent results from a study by Bathke et $a .^{3}$ are shown in Fig. 13. Figures 13(a) and 13(b) plot $\hat{I}_{\phi} /\left\langle I_{\phi}\right\rangle$ vs $\hat{\Phi} /\langle\Phi\rangle$ and $\hat{v}_{\phi} /\left\langle I_{\phi} R_{p}\right\rangle$ vs $\hat{\Phi} /\langle\Phi\rangle$, respectively, for a steady-state 18.5-MA Compact RFP Reactor (CRFPR). Here, $\hat{\mathrm{I}}_{\phi} /\left\langle\mathrm{I}_{\phi}\right\rangle$ denotes the ac toroldal current swing normalized to the mean toroldal current, $\hat{v}_{\phi} /\left\langle I_{\phi} R_{p}\right\rangle$ denotes the ac toroldal voltage normalized to the mean resistive component of the toroidal loop voltage, and $\hat{\Phi} /\langle\Phi\rangle$ represents the ac toroldal flux swing normalized to the mean toroldal flux. The curves are generated by substituting the ac voltages $\left(v_{\theta}\right.$ and $\left.v_{\phi}\right)$ in Eq. (43) and iterating their amplitudes until $\left\langle\dot{I}_{\phi}\right\rangle=0$. Tle driving frequency is set equal to $50 \mathrm{~Hz}$, which is a reasonable power grid value. In each figure, the two curves represent solutions based on both the Taylor-state Bessel-function model (BFM) and the modified Bessel function model (MBFM) adjusted for a reactor relevant $\beta$ of 0.23 . An overaIl summary of the current-drive system for the CRFPR. is listed in Table I (Ref. 3). An examination of Fig. 13 elucidates several important features of current drive by $F-\theta$ pumping. First, steady-state current drive is viable over a wide range of $\hat{v}_{\phi}$ and $\hat{v}_{\theta}$ or equivalently $\hat{v}_{\phi} /\left\langle I_{\phi} R_{p}\right\rangle$ and $\hat{\Phi} /\langle\Phi\rangle$ parameter space. This implies that the exact operating point in $\hat{v}_{\phi}, \hat{v}_{\theta}$ space can be determined by physics and engineering constraints in order to maximize the system efficiency and minimize its effect on reactor plasma properties.

Second, the current-drive effect is relatively insensitive to the exact RFP field model employed, as long as there exists a locus in F- $\theta$ space on which the relaxed states 11e. For example, Fig. 13(c) 1llustrates the $F-\theta$ trajectories for both the BFM and MBFM $(\beta=0.23)$. These substantially different models result in a modest $(\Sigma 50 \%)$ difference between the ac modulation requirements. 

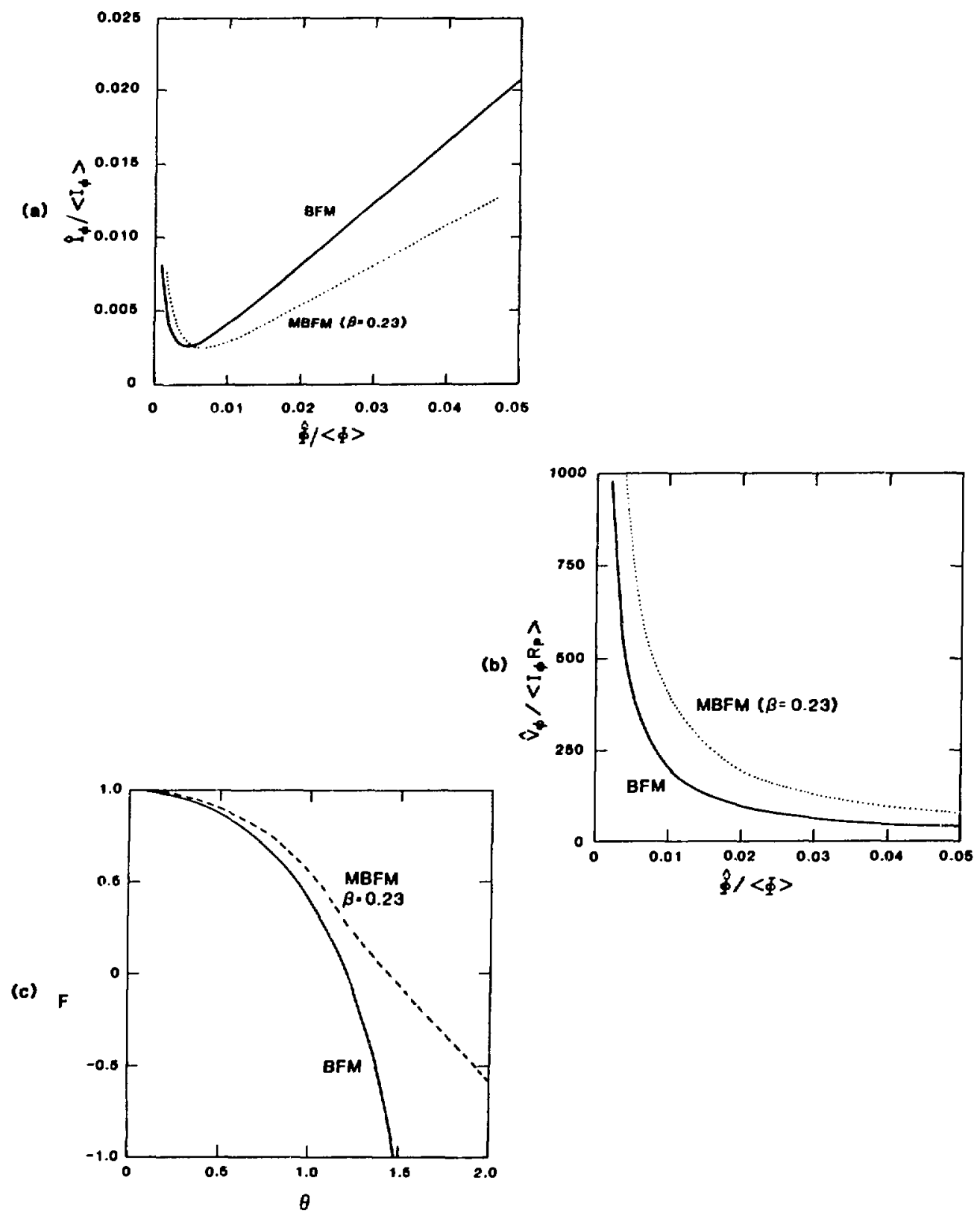

Fig. 13.

Computational study of $F-\Theta$ pumping on a Compact RFP Reactor (CRFPR). The system is driven at $50 \mathrm{~Hz}$ and optimum phase. The solld curves assume the $B F M$ while the dotted curves assume the MBFM corrected for a $\beta$ of 0.23: (a) $\hat{I}_{\phi} /\left\langle I_{\phi}\right\rangle$ vs $\hat{\Phi} /\langle\Phi\rangle$, (b) $\hat{v}_{\phi} /\left\langle I_{\phi} R_{p}\right\rangle$ vs $\hat{\Phi} /\langle\Phi\rangle$, and (c) $F-\theta$ trajectory for the BFM and MBFM $(\beta=0.23)$. (Fror Bathke et al., Ref. 3.) 
TABLE I

TYPICAI ( $F-\Theta$ PUMPING)

CURRENT-DRIVE CRFPR PARAMETERS

\begin{tabular}{ll}
\multicolumn{1}{c}{ PARAMETER } & VALUE \\
\hline Fractional Flux Swing $\hat{\phi} /\langle\phi\rangle$ & 0.01 \\
Normalized Voltage Sring, $\hat{v}_{\phi} /\left\langle I_{\phi} R_{p}\right\rangle$ & 200 \\
Toroidal voitage, $\left\langle I_{\phi} R_{p}\right\rangle(\mathrm{V})$ & 0.31 \\
Normalized Current Swing, $\hat{\mathrm{I}}_{\phi} /\left\langle\mathrm{I}_{\phi}\right\rangle$ & 0.004 \\
Current Swing, $\hat{\mathrm{I}}_{\phi}(\mathrm{MA})$ & 0.078 \\
Frequency, $\omega(\mathrm{Hz})$ & 50 \\
Dissipative Puwer, $\langle\mathrm{P}\rangle(\mathrm{MW})$ & 5.74 \\
$\quad$ Poloidal, $\left\langle\mathrm{P}_{\theta}\right\rangle(\mathrm{MW})$ & 3.44 \\
Toroidal, $\left\langle\mathrm{P}_{\phi}\right\rangle(\mathrm{MW})$ & 2.30 \\
Efficiency Factor, $\left\langle\mathrm{I}_{\phi}\right\rangle /\langle\mathrm{P}\rangle(\mathrm{A} / \mathrm{W})$ & 3.2 \\
Stored Energy, $\mathrm{E}_{\mathbf{s}}(\mathrm{MJ})$ & 0.26
\end{tabular}

Finally, with the expected low plasma resistivity of an ignited reactor, the power requirements and concomitant voltage and current modulation amplitudes for c. irent drive are low. For example, the design point for the F- $\Theta$ pumping system a the Compact RFP Reactor ${ }^{3}$ requires only $\langle\mathrm{P}\rangle=6 \mathrm{MW}$ of power (Table 1 ). This gives a current-drive efficiency of $\left\langle\tau_{\phi}\right\rangle /\langle P\rangle=3.2$ amperes per watt, more than an order of magnitude larger than the 0.2 value quoted as probably attainable for rf current drive assuming Tokamak reactor parameters.15 Modulation-Induced plasma motion has been estimated by $\underset{\sim}{\mathbf{E}} \times \underset{\sim}{\mathrm{B}}$ drift arguments and time-dependent equilitrium modelirg to be less than one per cent of the minor radius.

VII. F- $\Theta$ PUMPING AND THE DYNAMO EFFECT

One plausible explanation for the strong, nonlinear coupling and concomitant $F-\Theta$ pumping $1 \mathrm{n}$ an RFP is the existence of a "plasma dynamo." In one model the dynamo maintains the RFP mean field topology in a relaxed state by a process of field-line reconnection and plasma turbulence. That is, the plasma internally creates toroidal flux in order to maintain itself on the F- $\theta$ curve. Observation of self-reversal in past RFP experiments and recent work on $2 \mathrm{~T}-40 \mathrm{M}$ with multimillisecond-current risetimes substantiate the flux generction 
effert.16,17 0ther possibilities include a tangled discharge model 18 and a kinetic model 19 of RFP sustainment in which magnetic field stochasticity allows electron currents to be driven against the applied electric field in the reversed field region thus malntaining the RFP profiles. Although a comprehensive treatment of dynamo physics is beyond the scope of this paper, an introduction to one possible dynamo model, using the $\alpha$-effect, ${ }^{8}$ is given below. However, it must be emphasized that $F-\theta$ pumping relles only on the existence of a nonlinear plasma coupling and not on the particular nature of the physical coupling mechanism.

A. Simple Ohm's Law Model

A simple form of Ohm's law is given by

$$
\underset{\sim}{\mathrm{E}}+\underline{\sim} \times \underset{\mathrm{B}}{=}=\vec{\eta} \cdot \underset{\sim}{\mathrm{J}},
$$

where $\underset{\sim}{u}(\underset{x}{x}, t)$ denotes the velocity fleld of the plasma and $\bar{\eta}$ represents the plasma resistivity tensor. Applying Faraday's and Ohm's laws to any closed plasma magnetic field line yields

$$
\oint_{\ell} \underset{\sim}{\mathrm{E}} \cdot \mathrm{d} \ell=\oint \ddot{\eta} \cdot \underset{\sim}{J} \cdot \mathrm{d} \ell=-\dot{\psi}_{\mathrm{g}},
$$

where $\psi_{\mathbf{g}}$ denotes a generalized magnetic flux.

For an RFP, Eq. (57) is easily evaluated in terms of the orthogonal components $\left(\mathrm{E}_{\theta}, \mathrm{E}_{\phi}\right)$ by choosing the integration path along the toroidal fleld null (in the $\theta$ direction) and magnetic axis (in the $\phi$ direction), respectively. For these cases,

$$
\left.\mathrm{E}_{\theta}\right|_{\mathrm{nu} 11}=\frac{1}{\ell_{1}} \oint \eta_{\|} \mathrm{J}_{\theta} \mathrm{d} \ell_{1}=-\dot{\Phi} / \ell_{1}
$$

and

$$
\left.E_{\phi}\right\rfloor_{\text {axis }}=\frac{1}{\ell_{2}} \oint \eta_{\|} J_{\phi} \mathrm{d} \ell_{2}=-\dot{\psi} / \ell_{2},
$$


where $\Phi$ represents the toroidal magnetic flux enclosed by the path of length $l_{1}$ along the toroldal field null and $\psi$ denotes the total flux enclosed by the magnet1c axis of length $\ell_{2}$. For a constant current steady-state operation, $\dot{\psi}$ includes the monotonically changing magnetic flux through the central torus hole. Thus, the constraint that $\psi$ remain finite limits the present inductively driven experiments to a pulsed mode of operation.

Finite positive resistivity rules out a constant steady toroidal flux in the plasma becausa Eq. (58) Implies $\Phi$ must decay.17 There are two possible ways to circumvent this decay and explain the long ( $30 \mathrm{~ms}$ ) constant current pulsee observed on ZT-40M: (1) Ohm's law may not be valld and/or (2) the discharge may not be symmetric. The tangled discharge model retaine a local Ohm's law and gives up local field symmetry while the kinetic model abandons Ohm's law and local field symmetry. Both models can retaln symmetric space-averaged mean flelds.18,19 The turbulent dynamo retains a local ohm's law and gives up symmetry for the local instantaneous flelds but retains symmetry and has an altered Ohm's law for the mean fields.

\section{B. The Turbulent Dynamo}

In general, the magnetic and velocity $u$ flelds of an RFP plasma may be expressed in terms of a mean and fluctuating component, represented by \langle\rangle and $\delta$ symbols respectively,

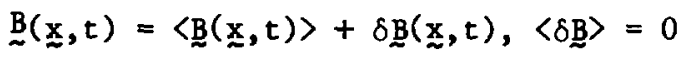

and

$$
\underline{u}(\underline{x}, t)=\langle\underline{u}(\underline{x}, t)\rangle+\delta \underline{u}(\underline{x}, t),\langle\delta \underline{u}\rangle=0
$$

Thus

$$
\langle\underline{\sim} \mathbf{x} \underset{\sim}{\mathrm{B}}\rangle=\langle\underline{\sim}\rangle \mathbf{x}\langle\underline{\sim}\rangle+\langle\delta \underline{\underline{u}} \mathbf{x} \delta \underline{\mathrm{B}}\rangle .
$$

Using Eq. (62), the mean value of Ohm's law [Eq. (56)] may be written as

$$
\langle\underline{\mathrm{E}}\rangle=\langle\vec{\eta} \cdot \underline{\mathrm{J}}\rangle-\langle\underline{\mathrm{u}}\rangle \times\langle\underline{\mathrm{B}}\rangle-\langle\delta \underline{\mathrm{u}} \times \delta \underline{\mathrm{B}}\rangle,
$$


where $\langle\underline{\sim}\rangle$ is the mean electric field in the laboratory frame of reference. With

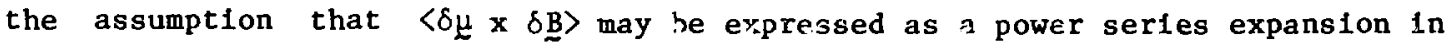
$\langle B\rangle$, then to lowest order for $\varepsilon_{\mathfrak{u}}$ isotropic, 8 one may write

$$
\langle\delta \underset{\sim}{\underline{u}} \mathbf{x} \delta \underset{\sim}{B}\rangle=\alpha\langle\underset{\sim}{\mathrm{B}}\rangle,
$$

where $\alpha$ is a pseudoscalar function of space and time. The mean-valued Ohm's law becomes

$$
\langle\underset{\sim}{\mathrm{E}}\rangle=\langle\vec{\eta} \cdot \mathrm{J}\rangle-\langle\underline{\sim}\rangle \times\langle\underline{\mathrm{B}}\rangle-\alpha\langle\underline{\mathrm{B}}\rangle \text {. }
$$

The appearance of the last term in the mean-fleld Ohm's law is called the $\alpha$ affect. For positive $\alpha$, it is now possible for $\langle E\rangle$ to vanish even though $\mathrm{J}$ remains finite. Hence, by utilizing the $\alpha$ effect dynamo, it is possible to sustain a steady-state mean p?.asma current. From Eq. (59), and for a steady state where $\langle\underline{u}\rangle=0$,

$$
\left\langle\mathrm{E}_{\phi}\right\rangle=\langle\vec{\eta} \cdot \underset{\sim}{\mathrm{J}}\rangle_{\|}-\alpha\langle\underset{\mathrm{B}}{ }\rangle=-\langle\dot{\psi}\rangle / \ell_{2},
$$

where \| denotes the component parallel to $\langle B\rangle$. Note that $\left\langle E_{\phi}\right\rangle$ can vanish if the two terms cance1. In this case, a mean $J_{\phi}$ and hence $\left\langle I_{\phi}\right\rangle$ can be maintained with $\langle\dot{\psi}\rangle=0$.

VIII. CONCLUSIONS

$F-\Theta$ pumping is at present a plausible and reactor-relevant candidate for RFP current drive. Analytic and computational studies indicate that F- $\theta$ pumping is relatively insensitive to RFP plasma models as long as the plasma's dynamic response to fleld modulation remains along a well-defined trajectory in $F-\theta$ space and the modulation does not significantly degrade overall pinch performance. Recent experimental measurements on the ZT-40M experiment have tentatively confirmed these conditions. Although many aspects of F- $\theta$ pumping must yet be tested by experiment, $F-\Theta$ pumping may provide a technologically simple method for driving future steady-state RFP devices. 
IX. ACKNOWLEDGMENTS

We acknowledge the entire Los Alamos experimental RFP team for its contributions in the experimental operation and measurements on $\mathrm{ZT}-40 \mathrm{M}$, which are used in this paper.

\section{REFERENCES}

1. N. J. Fisch, Princeton Plasma Physics Laboratory report PPPL-1772 (1981).

2. M. K. Bevir and J. W. Gray, in Proceedings of the Reversed F'eld Pinch Theory Workshop, Los Alamos National Laboratory report LA-8944-C, 176 (January 1981).

3. C. G. Bathke, M. J. Embrechts, R. L. Hagenson, R. A. Krakowsk1, and R. L. Miller, "Plasma Engineering Design of a Compact Reversed Field Pinch Reactor (CRFPR)," Los Alamos National Laboratory document LA-UR-83-3505, to be published in the Proceedings of the loth Symposium on Fusion Engineering, Philadelphia, 1983.

4. H. A. B. Bodin and A. A. Newton, Nucl. Fission 20, 1255 (1980).

5. D. A. Baker and W. E. Quinn, "The Reversed-Field Pinch," Fusion, Vol. I, Part A, E. Teller, Ed. (Academic Press, Inc., New York, 198ד Chap. 7.

6. J. B. Taylor, Phys, Rev. Lett. 33, 139 (1974).

7. K. F. Schoenberg, R. F. Gribble, and J. A. Phillips, Nucl. Fusion 22, 1433 (1982).

8. H. K. Moffatt, Magnetic Fleld veneration in Electrically Conducting Fluids (Cambridge University Press, Cambridge, Massachusetts, 1978).

9. L. Turner and J. P. Christiansen, Phys. Fluids 24, 893 (1981).

10. J. W. Johnston, Plasma Phys. 23, 187 (1981).

11. K. F. Schoenberg, R. W. Moses, and R. L. Hagenson, "Plasma Resistivity in the Presence of a Reversed Field Pinch Dynamo," Los Alamos National Laboratory document LA-UR-83-1739, to be published Phys. Fluids, July 1984.

12. K. F. Schoenberg and R. F. Gribble, "A Possible Steady-State Current Drive for Reversed Field Pinch Discharges," in Proceedings of the 9th Symposium on Fusion Engineering Research, IEEE Pub. No. 81CH 1715-2 NPS, 1923 (1981).

13. Kurt F. Schoenberg, Don A. Baker, Robert F. Gribble, and William Keass, "Oscillating Field Current Drive for Reversed Field Pinch Discharges," 1982 IEEE International Conference on Plasma Sclence, IEEE 82CH1770-7, 51 (1982). 